\title{
The optimal system for series systems with warm standby components and a repairable service station
}

\author{
M. Salah EL-Sherbeny \\ Helwan University \\ Department of Mathematics \\ Faculty of Science, Cairo \\ Egypt \\ P. O. Box 11795 \\ m_el_sherbeny@yahoo.com
}

\author{
A. M. Rashad \\ Helwan University \\ Department of Mathematics \\ Faculty of Science, Cairo \\ Egypt \\ P. O. Box 11795 \\ armoussa@hotmail.com \\ D. M. Gharieb \\ Helwan University \\ Department of Mathematics \\ Faculty of Science, Cairo \\ Egypt \\ P. O. Box 11795 \\ dina_gharieb1983@yahoo.com
}

\begin{abstract}
This paper deals with the reliability and availability characteristics of three different series system configurations with warm standby components and a repairable service station. The failure time of the primary and warm standby are assumed to be exponentially distributed with parameters $\lambda$ and $\alpha$ respectively. The repair time distribution of each server is also exponentially distributed with parameter $\mu$. The breakdown time and the repair time of the service station are also assumed exponentially distributed with parameters $\gamma$ and $\beta$ respectively. We derive the reliability dependent on time, availability dependent on time, the mean time to failure, $M T T F_{i}$, and the steady-state availability $A_{i}(\infty)$ for three configurations and perform comparisons. Comparisons are made for specific values of distribution parameters and of the cost of the components. The three configurations are ranked based on: $\operatorname{MTTF}_{i}, A_{i}(\infty)$ and $C_{i} / B_{i}$ where $B_{i}$ is either $M T T F_{i}$ or $A_{i}(\infty)$.
\end{abstract}

Keywords: reliability dependent on time, availability dependent on time, mean time to failure, steady-state availability, warm standby, cost/ benefit, repairable service station. 


\section{Introduction}

Redundancy, repair maintenance, and preventive maintenance are some of the well-known methods by which the reliability of a system can be improved. Twounit standby redundant systems have been extensively studied by several authors in the past. Achieving high reliability and /or availability is often an essential requisite.

In this paper, we consider the manufacturing system or the electric power systems (power plant) to be a serial system with standby components and repairable service station. A standby component called a "warm standby" when the failure rate is not equal zero and is less than the failure rate of a primary component. Primary and warm standby components can be considered to be repairable. We study three different series system configurations with warm standby components and a repairable service station. These three configurations are compared based on their mean time-to-failure, MTTF, their steady-state availability, $A(\infty)$, and their cost/benefit ratio $C / B$. Cost is considered to be a size-proportional cost for the components. Benefit is divided into two categories according to whether the measure used is the system reliability given by MTTF or the system availability given by $A(\infty)$.

Therefore, Wang and Sivazlian [4] compared the two different configurations with parallel components based on their overall availability and life cycle costs under uncertainty in systems lifetime. The time-to-repair and the time-to-failure for each of the primary and parallel components are assumed to have the negative exponential distribution. Wang and Pearn [5] studied the cost benefit analysis of series systems with warm standby components. They suggested the time-to-repair and the time-to-failure for each of the primary and warm standby components is assumed to have the negative exponential distribution. El-Said and El-Sherbeny [3] studied two systems each system with two parallel components, the second system differs from the first system due to the additional feature of preventive maintenance. The two systems are analyzed under the assumption that the failure, replacement and preventive maintenance times of the units are assumed to be arbitrarily distributed. Wang et.al [7] considered four different system configurations with warm standby components and standby switching failures are compared based on their reliability and availability, when the time-to-repair and the time-to-failure for each of the primary and warm standby components are assumed to follow the negative exponential distribution. Chandrasekhar et al. [1] studied the two unit standby system and obtain exact confidence limits for the steady-state availability of the system, when the failure rate of an operative unit is constant and the repair time of the failed unit is a two stage Erlang distribution. Dhillon and Raypati [2] considered the stochastic analysis of two-unit outdoor electric power systems in changing weather. Wang et.al [6] studies the cost benefit analysis of series systems with cold standby components and a repairable service station, when the service times and the failure times of the primary components are assumed exponentially distributed. The breakdown times and the repair times of the service station are 
also assumed exponentially distributed. The purpose of this paper is accomplishes three objectives. The first one is to use Laplace transform techniques to derive the explicit expressions for the mean time-to-failure $\operatorname{MTTF}_{i}$ and the steady-state availability $A_{i}(\infty)$ for configuration $i(i=1,2,3)$. The second one is to compare these three configurations in terms of their $M T T F_{i}$ and their $A_{i}(\infty)$. The last one is to compare the three configurations with their cost/benefit $\left(C_{i} / B_{i}\right)$ based on assumed numerical values given to the distribution parameters, as well as to the costs for the components.

\section{Description of the system}

The present paper is devoted to consider the requirements of a $10 \mathrm{MW}$ power plant. We assume that generators are available in units of both 10 and $5 \mathrm{MW}$. We also assume that standby generators are allowed to fail while inactive before they are put into full operation, and that the standby generators are continuously monitored by a fault detecting device in order to identify if they fail or not. Let us assume that all switchover times are instantaneous and switching is perfect. Primary components and warm standby components can be considered to be repairable. Each of the primary components fails independently of the state of the others and has an exponential failure distribution with parameter $\lambda$. Whenever one of these primary components fails, it is immediately replaced by a standby component if one is available. We assume that each of the available standby components fails independently of the state of all the others and has an exponential failure distribution with parameter $\alpha$ where $\alpha \in(0, \lambda)$. Whenever a primary component or a standby component fails, it is immediately sent to a service station where it is served in order of breakdowns, with identical service rate $\mu$. Once a component is served, it is as good as new. There is a single service station which may be breakdown only when the service station is serving a component. Once the service station breaks down, the service station enters a breakdown state and a failed component must wait until the service station is repaired. The service station has an exponential failure distribution with rate $\gamma$. Whenever a service station breaks down, it is immediately repaired at a repair rate $\beta$. Repair time distribution of the service station is assumed to be exponentially distributed. Service is allowed to be interrupted if the station breaks down, and the station is immediately repaired. As soon as the repair of a service station is completed, the service station enters a working state and continues to serve a failed component immediately. After the service station is repaired, it is as good as new. Further, failure times and repair times are independently distributed random variables. The following configurations are considered. The first configuration is a serial system of one primary $10 \mathrm{MW}$ component with two interchangeable warm standby $10 \mathrm{MW}$ components. The second configuration is a serial system of two primary $5 \mathrm{MW}$ components and one warm standby $5 \mathrm{MW}$ component. The last configuration is a serial system of two primary $5 \mathrm{MW}$ components with two interchangeable warm standby $5 \mathrm{MW}$ components. Each 
standby unit can replace either one of the failed components. If necessary, a standby unit can also replace the first used standby unit in case of failure.

\section{Cost-Benefit Ratio}

The size-proportional costs for the primary and warm standby components are given in Table 1. Thus we calculate the costs for each configuration $i(i=1,2,3)$ as shown in Table 2. Let $C_{i}$ denote the cost of the configuration $i$, and $B_{i}$ be the benefit of the configuration $i$, where $B_{i}$ may either be the $\operatorname{MTTF}_{i}$ ( Mean time to system failure), or the $A_{i}(\infty)$ (Steady-state availability), where $i=1,2,3$.

\begin{tabular}{|cc|}
\hline Component & Cost (in\$) \\
\hline Primary 10 MW & $10 \times 10^{6}$ \\
Primary 5 MW & $5 \times 10^{6}$ \\
Warm standby 10 MW & $6 \times 10^{6}$ \\
Warm standby 5 MW & $3 \times 10^{6}$ \\
\hline
\end{tabular}

Table 1.

The size-proportional cost for the primary and warm standby components

\begin{tabular}{|c|c|}
\hline Configuration & Cost (in \$) \\
\hline Configuration 1 & $22 \times 10^{6}$ \\
\hline Configuration 2 & $13 \times 10^{6}$ \\
\hline Configuration 3 & $16 \times 10^{6}$ \\
\hline
\end{tabular}

Table 2.

we defined

The costs for each configuration $i \forall i=1,2,3$.

$$
\begin{array}{cccc}
h_{1}=\lambda+2 \alpha, & h_{2}=\lambda+\mu+\gamma, & h_{3}=\lambda+\beta, & h_{4}=\lambda+\alpha, \\
h_{5}=\beta+\gamma, & h_{6}=\alpha+\beta, & h_{7}=2 \alpha+\beta, & h_{8}=2 \lambda+\alpha, \\
h_{9}=2 \lambda+\mu+\gamma, & h_{10}=2 \lambda+\beta, & h_{11}=2 \lambda+2 \alpha . &
\end{array}
$$

\section{Problem solutions}

\subsection{Calculations for configuration 1}

\subsubsection{Reliability}

For configuration 1 , let $p_{n}(t)$ be the probability that exactly $n$ components are working at time $\mathrm{t}$ in the system when the service station is up and $Q_{n}(t)$ be the probability that exactly $\mathrm{n}$ components are working at time $\mathrm{t}$ in the system when the service station is broken down, where $(t \geq 0)$. The system reliability $R_{1}(t)$ is the probability of failure-free operation of the system in $(0, t]$. To derive an expression for the reliability of the system, we restrict the transitions of the 
Markov process to the up states. The initial conditions for configuration 1 are given by $p_{3}(0)=1, q_{3}(0)=0$ and $p_{k}(0)=q_{k}(0)=0 \quad \forall k=1,2$.

Thus the differential-difference equations are obtained:

$$
\begin{aligned}
& p_{3}^{\prime}(t)=-\left[h_{1}+\gamma\right] p_{3}(t)+\mu p_{2}(t)+\beta q_{3}(t), \\
& p_{2}^{\prime}(t)=-\left[h_{2}+\alpha\right] p_{2}(t)+\mu p_{1}(t)+h_{1} p_{3}(t)+\beta q_{2}(t), \\
& p_{1}^{\prime}(t)=-h_{2} p_{1}(t)+h_{4} p_{2}(t)+\beta q_{1}(t) \\
& q_{3}^{\prime}(t)=-\left[h_{1}+\beta\right] q_{3}(t)+\gamma p_{3}(t) \\
& q_{2}^{\prime}(t)=-\left[h_{3}+\alpha\right] q_{2}(t)+\gamma p_{2}(t)+h_{1} q_{3}(t), \\
& q_{1}^{\prime}(t)=-h_{3} q_{1}(t)+\gamma p_{1}(t)+h_{4} q_{2}(t) .
\end{aligned}
$$

Let $L_{p_{i}}(s), L_{q_{i}}(s)$ be the Laplace transform of $p_{i}(t), q_{i}(t) \forall i=1,2,3$. Taking Laplace transform on both the sides of the differential equations given above, solving for $L_{p_{i}}(s), L_{q_{i}}(s) \forall i=1,2,3$ and inverting, we get $p_{i}(t)$ and $q_{i}(t)$. Then the system reliability is given by.

$$
\begin{aligned}
R_{1}(t) & =\sum_{i=1}^{3} p_{i}(t)+\sum_{i=1}^{3} q_{i}(t) \\
& =\sum_{i=0}^{5} \frac{H\left(s_{i}\right)}{\prod_{\substack{j=0 \\
i \neq j}}^{5}\left(s_{i}-s_{j}\right)} \exp \left(s_{i} t\right)
\end{aligned}
$$

where,

$s_{0}, s_{1}, s_{2}, s_{3}, s_{4}$ and $s_{5}$ are the roots of the following equation:

$$
\left[\begin{array}{cccccc}
s+h_{1}+\gamma & -\mu & 0 & -\beta & 0 & 0 \\
-h_{1} & s+h_{2}+\alpha & -\mu & 0 & -\beta & 0 \\
0 & -h_{4} & s+h_{2} & 0 & 0 & -\beta \\
-\gamma & 0 & 0 & s+h_{1}+\beta & 0 & 0 \\
0 & -\gamma & 0 & -h_{1} & s+h_{3}+\alpha & 0 \\
0 & 0 & -\gamma & 0 & -h_{4} & s+h_{3}
\end{array}\right]=0
$$

and, $H\left(s_{i}\right)$ ( see in the appendix).

The mean time to failure $\left(M T T F_{1}\right)$ is given by

$M T T F_{1}=\int_{0}^{\infty} R_{1}(t) d t=\lim _{s \rightarrow 0}\left[\sum_{i=1}^{3} P_{i}(s)+\sum_{i=1}^{3} Q_{i}(s)\right]$. 
For configuration 1, we get the following explicit expression for the $M T T F_{1}$ :

$$
\text { MTTF }_{1}=\frac{H\left(s_{i}=0\right)}{h_{1} h_{4} \lambda\left(\left(h_{1}+h_{5}\right)\left(h_{3}+\gamma\right)\left(h_{3}+\alpha+\gamma\right)+2 \gamma\left(h_{3}+\alpha+\gamma\right) \mu+\gamma \mu^{2}\right)}
$$

\subsubsection{System availability}

The system availability is the probability that the system operates within the tolerances at a given instant of time and is obtained as follows:

$$
\begin{aligned}
& p_{3}^{\prime}(t)=-\left[h_{1}+\gamma\right] p_{3}(t)+\mu p_{2}(t)+\beta q_{3}(t), \\
& p_{2}^{\prime}(t)=-\left[h_{2}+\alpha\right] p_{2}(t)+h_{1} p_{3}(t)+\mu p_{1}(t)+\beta q_{2}(t), \\
& p_{1}^{\prime}(t)=-h_{2} p_{1}(t)+h_{4} p_{2}(t)+\mu p_{0}(t)+\beta q_{1}(t) \\
& p_{0}^{\prime}(t)=-[\mu+\gamma] p_{0}(t)+\lambda p_{1}(t)+\beta q_{0}(t), \\
& q_{3}^{\prime}(t)=-\left[h_{1}+\beta\right] q_{3}(t)+\gamma p_{3}(t) \\
& q_{2}^{\prime}(t)=-\left[h_{3}+\alpha\right] q_{2}(t)+\gamma p_{2}(t)+h_{1} q_{3}(t) \\
& q_{1}^{\prime}(t)=-h_{3} q_{1}(t)+\gamma p_{1}(t)+h_{4} q_{2}(t) \\
& q_{0}^{\prime}(t)=-\beta q_{0}(t)+\gamma p_{0}(t)+\lambda q_{1}(t)
\end{aligned}
$$

We solve equations $(9-16)$ by using $\sum_{i=0}^{3} p_{i}(t)+\sum_{i=0}^{3} q_{i}(t)=1$. Taking Laplace transform on both the sides of the differential equations given above, solving for $L_{p_{i}}(s), L_{q_{i}}(s), i=0,1,2,3$ and inverting, we get $p_{i}(t), q_{i}(t) ; i=0,1,2,3$. Then the system availability is given by.

$$
A_{1}(t)=\frac{H_{1}}{\prod_{i=0}^{5} s_{i}}+\sum_{i=0}^{5} \frac{H_{1}\left(s_{i}\right)}{\prod_{\substack{j=0 \\ i \neq j}}^{5} s_{i}\left(s_{i}-s_{j}\right)} \exp \left(s_{i} t\right)
$$

where,

$$
\begin{aligned}
& \prod_{i=0}^{5} s_{i}=\left[h _ { 5 } \left(h_{1} h_{4}\left(h_{1}+h_{5}\right)\left(h_{3}+\gamma\right)\left(h_{3}+\alpha+\gamma\right) \lambda+h_{1} h_{4}\left(h_{3}+\alpha+\gamma\right)\left(h_{5} \beta+2 \alpha \beta+\lambda\left(2 h_{6}+\right.\right.\right.\right. \\
& 3 \gamma+\lambda)) \mu+h_{1}\left(h_{7} \alpha \beta+h_{5}\left(h_{7} \beta+3 h_{3} \lambda\right)+\lambda\left(2 \alpha^{2}+\lambda^{2}+3 \alpha(2 \beta+\gamma+\lambda)\right)\right) \mu^{2}+h_{3}\left(h_{3}+\right. \\
& \left.\left.\alpha)\left(h_{1}+\beta\right) \mu^{3}\right)\right],
\end{aligned}
$$

where $H_{1}$ and $H_{1}\left(s_{i}\right)$ are shown in appendix.

For configuration 1, the explicit expression for the $A_{1}(\infty)$ is given by

$$
A_{1}(\infty)=\frac{H_{1}}{\prod_{i=0}^{5} s_{i}}
$$




\subsection{Calculations for configuration 2}

\subsubsection{Reliability}

For configuration 2 are given by $p_{3}(0)=1, p_{2}(0)=0$ and $q_{k}(0)=0 \quad \forall k=2,3$. The system of differential-difference equations associated with configuration 2 is:

$p_{3}^{\prime}(t)=-\left[h_{8}+\gamma\right] p_{3}(t)+\mu p_{2}(t)+\beta q_{3}(t)$,

$p_{2}^{\prime}(t)=-h_{9} p_{2}(t)+h_{8} p_{3}(t)+\beta q_{2}(t)$,

$q_{3}^{\prime}(t)=-\left[h_{8}+\beta\right] q_{3}(t)+\gamma p_{3}(t)$,

$q_{2}^{\prime}(t)=-h_{10} q_{2}(t)+\gamma p_{2}(t)+h_{8} q_{3}(t)$

Taking Laplace transform on both the sides of the differential equations given above, solving for $L_{p_{i}}(s), L_{q_{i}}(s) \quad \forall i=2,3$ and inverting, we get $p_{i}(t), q_{i}(t) \forall i=2,3$. Then the system reliability is given by.

$R_{2}(t)=\sum_{i=0}^{3} \frac{E\left(s_{i}\right)}{\prod_{\substack{j=0 \\ i \neq j}}^{3}\left(s_{i}-s_{j}\right)} \exp \left(s_{i} t\right)$

where,

$s_{0}, s_{1}, s_{2}$ and $s_{3}$ are the roots of the following equation:

$$
\left[\begin{array}{cccc}
s+h_{8}+\gamma & -\mu & -\beta & 0 \\
-h_{8} & s+h_{9} & 0 & -\beta \\
-\gamma & 0 & s+h_{8}+\beta & 0 \\
0 & -\gamma & -h_{8} & s+h_{10}
\end{array}\right]=0
$$

and, $E\left(s_{i}\right)$ (see in the appendix).

The mean time to failure $\left(M_{T T F}\right)$ is given by

$M T T F_{2}=\int_{0}^{\infty} R_{2}(t) d t=\lim _{s \rightarrow 0}\left[\sum_{i=2}^{3} P_{i}(s)+\sum_{i=2}^{3} Q_{i}(s)\right]$.

For configuration 2, we get the following explicit expression for the $M T T F_{2}$ :

MTTF $_{2}=\frac{E\left(s_{i}=0\right)}{2 h_{8} \lambda\left(\left(h_{5}+h_{8}\right)\left(h_{10}+\gamma\right)+\gamma \mu\right)}$ 


\subsubsection{System availability}

For the availability case of configuration 2 , the differential equations can be expressed as:

$$
\begin{aligned}
& p_{3}^{\prime}(t)=-\left[h_{8}+\gamma\right] p_{3}(t)+\mu p_{2}(t)+\beta q_{3}(t), \\
& p_{2}^{\prime}(t)=-h_{9} p_{2}(t)+h_{8} p_{3}(t)+\mu p_{1}(t)+\beta q_{2}(t), \\
& p_{1}^{\prime}(t)=-[\mu+\gamma] p_{1}(t)+2 \lambda p_{2}(t)+\beta q_{1}(t), \\
& q_{3}^{\prime}(t)=-\left[h_{8}+\beta\right] q_{3}(t)+\gamma p_{3}(t), \\
& q_{2}^{\prime}(t)=-h_{10} q_{2}(t)+\gamma p_{2}(t)+h_{8} q_{3}(t), \\
& q_{1}^{\prime}(t)=-\beta q_{1}(t)+\gamma p_{1}(t)+2 \lambda q_{2}(t)
\end{aligned}
$$

Taking Laplace transform on both the sides of the differential equations given above, solving for $L_{p_{i}}(s), L_{q_{i}}(s) \quad \forall i=1,2,3$ and inverting, we get $p_{i}(t), q_{i}(t) \forall i=1,2,3$. Then the system availability is given by.

$$
A_{2}(t)=\frac{E_{1}}{\prod_{i=0}^{5} s_{i}}+\sum_{i=0}^{5} \frac{E_{1}\left(s_{i}\right)}{\prod_{\substack{j=0 \\ i \neq j}}^{5} s_{i}\left(s_{i}-s_{j}\right)} \exp \left(s_{i} t\right)
$$

where,

$\prod_{i=0}^{5} s_{i}=h_{5}\left(2 h_{8}\left(h_{5}+h_{8}\right) \lambda\left(h_{5}+2 \lambda\right)+h_{8}\left(\left(h_{5}+\alpha\right) \beta+2\left(2 h_{5}+\alpha\right) \lambda+4 \lambda^{2}\right) \mu+h_{10}\left(h_{8}+\beta\right) \mu^{2}\right)$, $E_{1}$ and $E_{1}\left(s_{i}\right)$ (see in the appendix).

For configuration 2, the explicit expression for the $A_{2}(\infty)$ is given by

$$
A_{2}(\infty)=\frac{E_{1}}{\prod_{i=0}^{5} s_{i}}
$$

\subsection{Calculations for configuration 3}

\subsubsection{Reliability}

For configuration 3 are given by $p_{4}(0)=1, q_{4}(0)=0$ and $q_{k}(0)=p_{k}(0)=0$ $\forall k=2,3$. The system of differential-difference equations associated with configuration 3 is:

$$
\begin{aligned}
& p_{4}^{\prime}(t)=-\left[h_{11}+\gamma\right] p_{4}(t)+\mu p_{3}(t)+\beta q_{4}(t), \\
& p_{3}^{\prime}(t)=-\left[h_{9}+\alpha\right] p_{3}(t)+h_{11} p_{4}(t)+\mu p_{2}(t)+\beta q_{3}(t), \\
& p_{2}^{\prime}(t)=-h_{9} p_{2}(t)+h_{8} p_{3}(t)+\beta q_{2}(t), \\
& q_{4}^{\prime}(t)=-\left[h_{11}+\beta\right] q_{4}(t)+\gamma p_{4}(t), \\
& q_{3}^{\prime}(t)=-\left[h_{8}+\beta\right] q_{3}(t)+\gamma p_{3}(t)+h_{11} q_{4}(t), \\
& q_{2}^{\prime}(t)=-h_{10} q_{2}(t)+\gamma p_{2}(t)+h_{8} q_{3}(t)
\end{aligned}
$$


Taking Laplace transform on both the sides of the differential equations given above, solving for $L_{p_{i}}(s), L_{q_{i}}(s), \quad i=2,3,4$ and inverting, we get $p_{i}(t), q_{i}(t) i=2,3,4$. Then the system reliability is given by.

$R_{3}(t)=\sum_{i=0}^{5} \frac{Z\left(s_{i}\right)}{\prod_{\substack{j=0 \\ i \neq j}}^{5}\left(s_{i}-s_{j}\right)} \exp \left(s_{i} t\right)$

where,

$s_{0}, s_{1}, s_{2}, s_{3}, s_{4}$ and $s_{5}$ are the roots of the following equation:

$\left[\begin{array}{cccccc}s+h_{11}+\gamma & -\mu & 0 & -\beta & 0 & 0 \\ -h_{11} & s+h_{9}+\alpha & -\mu & 0 & -\beta & 0 \\ 0 & -h_{8} & s+h_{9} & 0 & 0 & -\beta \\ -\gamma & 0 & 0 & s+h_{11}+\beta & 0 & 0 \\ 0 & -\gamma & 0 & -h_{11} & s+h_{8}+\beta & 0 \\ 0 & 0 & -\gamma & 0 & -h_{8} & s+h_{10}\end{array}\right]=0$,

and, $Z(s)$ ( see in the appendix ).

The mean time to failure $\left(M_{T T F}\right)$ is given by

$M T T F_{3}=\int_{0}^{\infty} R_{3}(t) d t=\lim _{s \rightarrow 0}\left[\sum_{i=2}^{4} P_{i}(s)+\sum_{i=2}^{4} Q_{i}(s)\right]$.

For configuration 3, we get the following explicit expression for the $M T T F_{3}$ :

MTTF $_{3}=\frac{Z\left(s_{i}=0\right)}{\left(4 h_{4} h_{8} \lambda\left(\left(h_{10}+\gamma\right)\left(h_{10}+\alpha+\gamma\right)\left(h_{10}+2 \alpha+\gamma\right)+2 \gamma\left(h_{10}+\alpha+\gamma\right) \mu\right)+\gamma \mu^{2}\right)}$

\subsubsection{System availability}

For the availability case of configuration 3 , the differential equations can be expressed as:

$p_{4}^{\prime}(t)=-\left[h_{11}+\gamma\right] p_{4}(t)+\mu p_{3}(t)+\beta q_{4}(t)$,

$p_{3}^{\prime}(t)=-\left[h_{9}+\alpha\right] p_{3}(t)+h_{11} p_{4}(t)+\mu p_{2}(t)+\beta q_{3}(t)$,

$p_{2}^{\prime}(t)=-h_{9} p_{2}(t)+h_{8} p_{3}(t)+\mu p_{1}(t)+\beta q_{2}(t)$,

$p_{1}^{\prime}(t)=-[\mu+\gamma] p_{1}(t)+2 \lambda p_{2}(t)+\beta q_{1}(t)$,

$q_{4}^{\prime}(t)=-\left[h_{11}+\beta\right] q_{4}(t)+\gamma p_{4}(t)$,

$q_{3}^{\prime}(t)=-\left[h_{8}+\beta\right] q_{3}(t)+\gamma p_{3}(t)+h_{11} q_{4}(t)$,

$q_{2}^{\prime}(t)=-h_{10} q_{2}(t)+\gamma p_{2}(t)+h_{8} q_{3}(t)$,

$q_{1}^{\prime}(t)=-\beta q_{1}(t)+\gamma p_{1}(t)+2 \lambda q_{2}(t)$ 
Taking Laplace transform on both the sides of the differential equations given above, solving for $L_{p_{i}}(s), L_{q_{i}}(s), \quad i=1,2,3,4$ and inverting, we get $p_{i}(t), q_{i}(t) i=1,2,3,4$. Then the system availability is given by.

$A_{3}(t)=\frac{Z_{1}}{\prod_{i=0}^{7} s_{i}}+\sum_{i=0}^{7} \frac{Z_{1}\left(s_{i}\right)}{\prod_{\substack{j=0 \\ i \neq j}}^{7} s_{i}\left(s_{i}-s_{j}\right)} \exp \left(s_{i} t\right)$

where,

$\prod_{i=0}^{7} s_{i}=h_{5}\left(4 h_{4}\left(h_{11}+h_{5}\right) h_{8}\left(h_{5}+h_{8}\right) \lambda\left(h_{5}+2 \lambda\right)+2 h_{4} h_{8}\left(h_{5}+h_{8}\right)\left(h_{5} \beta+2 \alpha \beta+2 \lambda\left(2 h_{6}\right.\right.\right.$

$+3 \gamma+2 \lambda)) \mu+2 h_{4}\left(h_{7}\left(h_{5}+\alpha\right) \beta+2\left(3 h_{5} \beta+\alpha(2 \alpha+6 \beta+3 \gamma)\right) \lambda+12\left(h_{5}+\alpha\right) \lambda^{2}+8 \lambda^{3}\right) \mu^{2}$

$\left.+h_{10}\left(h_{11}+\beta\right)\left(h_{8}+\beta\right) \mu^{3}\right)$,

where $Z_{1}$ and $Z_{1}\left(s_{i}\right)$ are shown in appendix.

For configuration 3 , the explicit expression for the $A_{3}(\infty)$ is given by $A_{3}(\infty)=\frac{Z_{1}}{\prod_{i=0}^{7} s_{i}}$

\section{Comparison of the three configuration}

The purpose of this section is to present specific comparisons for the $M T T F_{i}$ and the $A_{i}(\infty)$. Using an efficient Mathematica and Matlab computer program, all configurations will be compared in terms of their $M T T F_{i}$ and $A_{i}(\infty) \forall i=1,2,3$ with the following values.

$\frac{1}{\lambda}=1000$ days, $\frac{1}{\mu}=50$ days, $\frac{1}{\alpha}=1600$ days, $\frac{1}{\beta}=300$ days and $\frac{1}{\gamma}=2000$ days.

\subsection{Comparison for the $M T T F_{i}$ and $A_{i}(\infty)$}

We first consider the following five cases to perform a comparison for the $M T T F_{i}$ and $A_{i}(\infty)$ of the configurations $1,2,3$.

Case 1: We fix $\alpha=0.000625, \quad \mu=0.02, \beta=0.003, \gamma=0.0005$ and vary the values of $\lambda \in[0.001,0.002]$.

Case 2: We fix $\alpha=0.000625, \lambda=0.001, \beta=0.003, \gamma=0.0005$ and vary the values of $\mu \in[0.02,0.033]$. 
Case 3: We fix $\mu=0.02, \lambda=0.001, \beta=0.003, \gamma=0.0005$ and vary the values of $\alpha \in[0.000625,0.000833]$.

Case 4: We fix $\mu=0.02, \lambda=0.001, \alpha=0.000625, \gamma=0.0005$ and vary the values of $\beta \in[0.003,0.004]$.

Case 5: We fix $\mu=0.02, \lambda=0.001, \alpha=0.000625, \beta=0.003$ and vary the values of $\gamma \in[0.0005,0.0007]$.

Numerical results of $M T T F_{i}$ and $A_{i}(\infty)$ for configurations $\forall i=1,2,3$ are shown in Tables 3 and 4 for cases 1-5, respectively.

\subsection{Comparison of all configurations based on cost / benefit ratios}

In the above section, we did not consider that the different configurations may have different costs. However, these costs must be considered when comparing all configurations. The cost $\left(C_{i}\right)$ of the configuration $i \forall i=1,2,3$ are listed in table 2. Under the cost / benefit ratios, namely, $C_{i} / M T T F_{i}$ and $C_{i} / A_{i}(\infty)$, comparison are made based on assumed specific values given to the system parameters, and to the costs of the configurations.

Numerical results of $C_{i} / M T T F_{i}$ and $C_{i} / A_{i}(\infty)$ for configurations $\forall i=1,2,3$ are shown in Tables 5 and 6 for case $1-5$, respectively.

\begin{tabular}{|cc|}
\hline Range of $\lambda$ & Result \\
$0.001<\lambda<0.002$ & $M T T F_{1}>M T T F_{3}>M T T F_{2}$ \\
Range of $\mu$ & \\
$0.02<\mu<0.03$ & $M T T F_{1}>M T T F_{3}>M T T F_{2}$ \\
Range of $\alpha$ & \\
$0.000625<\alpha<0.000833$ & $M T T F_{1}>M T T F_{3}>M T T F_{2}$ \\
Range of $\beta$ & \\
$0.003<\beta<0.004$ & $M T T F_{1}>M T T F_{3}>M T T F_{2}$ \\
Range of $\gamma$ & \\
$0.0005<\gamma<0.0007$ & $M T T F_{1}>M T T F_{3}>M T T F_{2}$ \\
\hline
\end{tabular}

Table 3

Comparison of the configurations 1, 2, 3 for $M T T F_{i}$ 


\begin{tabular}{|cc|}
\hline Range of $\lambda$ & Result \\
$0.001<\lambda<0.002$ & $A_{1}(\infty)>A_{3}(\infty)>A_{2}(\infty)$ \\
Range of $\mu$ & \\
$0.02<\mu<0.03$ & $A_{1}(\infty)>A_{3}(\infty)>A_{2}(\infty)$ \\
Range of $\alpha$ & $A_{1}(\infty)>A_{3}(\infty)>A_{2}(\infty)$ \\
$0.000625<\alpha<0.000833$ & \\
Range of $\beta$ & $A_{1}(\infty)>A_{3}(\infty)>A_{2}(\infty)$ \\
$0.003<\beta<0.004$ & \\
Range of $\gamma$ & $A_{1}(\infty)>A_{3}(\infty)>A_{2}(\infty)$ \\
$0.0005<\gamma<0.0007$ &
\end{tabular}

Table 4

Comparison of the configurations 1, 2, 3 for $A_{i}(\infty)$

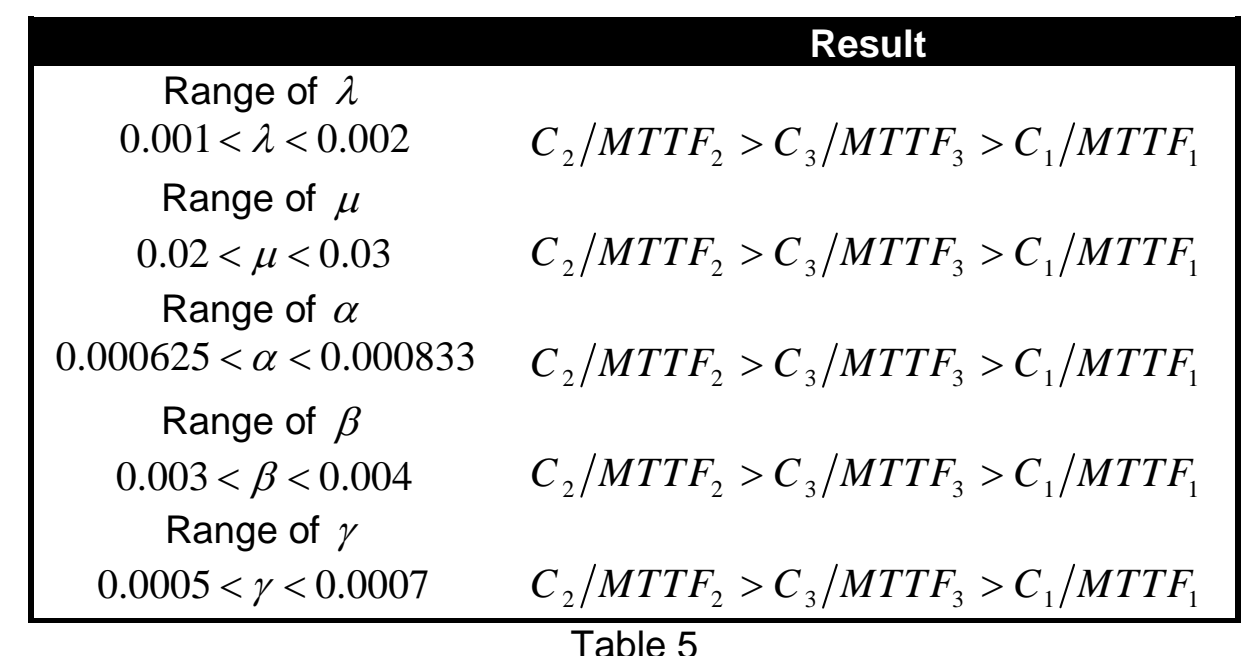

Comparison of the configurations $1,2,3$ for $C_{i} / M T T F_{i}$

\begin{tabular}{|cc|}
\hline Range of $\lambda$ & Result \\
$0.001<\lambda<0.002$ & $C_{1} / A_{1}(\infty)>C_{3} / A_{3}(\infty)>C_{2} / A_{2}(\infty)$ \\
Range of $\mu$ & \\
$0.02<\mu<0.03$ & $C_{1} / A_{1}(\infty)>C_{3} / A_{3}(\infty)>C_{2} / A_{2}(\infty)$ \\
Range of $\alpha$ & \\
$0.000625<\alpha<0.000833$ & $C_{1} / A_{1}(\infty)>C_{3} / A_{3}(\infty)>C_{2} / A_{2}(\infty)$ \\
Range of $\beta$ & \\
$0.003<\beta<0.004$ & $C_{1} / A_{1}(\infty)>C_{3} / A_{3}(\infty)>C_{2} / A_{2}(\infty)$ \\
Range of $\gamma$ & \\
$0.0005<\gamma<0.0007$ & $C_{1} / A_{1}(\infty)>C_{3} / A_{3}(\infty)>C_{2} / A_{2}(\infty)$ \\
\hline
\end{tabular}

Table 6

Comparison of the configurations $1,2,3$ for $C_{i} / A_{i}(\infty)$ 
The results of the cost $/ M T T F_{i}$ for each configurations $i \forall i=1,2,3$ are listed in table 5 for cases $1-5$, respectively. Table 5 reveal that the optimal configuration using $C_{i} / M_{T T F_{i}}$ value depends on the values of $\lambda, \alpha, \mu, \beta$ and $\gamma$. When $0.001<\lambda<0.002,0.02<\mu<0.03,0.000625<\alpha<0.000833,0.003<\beta<0.004$ and $0.0005<\gamma<0.0007$ the optimal configuration is configuration 1 .

The results of the cost $/ A_{i}(\infty)$ for each configurations $i \forall i=1,2,3$ are listed in table 6 for cases 1-5, respectively. Table 6 reveal that the best configuration using cost $/ A_{i}(\infty)$ value depends on the values of $\lambda, \alpha, \mu, \beta$ and $\gamma$. When $0.001<\lambda<0.002,0.02<\mu<0.03,0.000625<\alpha<0.000833,0.003<\beta<0.004$ and $0.0005<\gamma<0.0007$ the optimal configuration is configuration 2 .

\section{Special case}

[1] When $\alpha=0$ i.e "warm standby components convert to cold standby components". The optimal configuration using the cost/MTTF $F_{i}$ measure is configuration 1. Next, the optimal configuration using the $\operatorname{cost} / A_{i}(\infty)$ measure is configuration 2.

[2] When $\alpha=\lambda$ i.e "warm standby components convert to hot standby components". The optimal configuration using the cost/MTTF $F_{i}$ measure is configuration 1. Next, the optimal configuration using the $\operatorname{cost} / A_{i}(\infty)$ measure is configuration 2.

\section{Conclusions}

In this paper, we studied the mean time to system failure and the steady-state availability of three different series system configurations with warm standby components and a repairable service station. By comparing the MTTF and $A_{i}(\infty)$ listed in Tables $(3,4)$. Numerical results for the cost/benefit measure have been obtained for all configurations as in tables $(5,6)$. It is interesting to note first that the optimal configuration using the $\operatorname{cost} / M T T F_{i}$ measure is configuration 1. Next, the optimal configuration using the cost $/ A_{i}(\infty)$ measure is configuration 2.

\section{Appendix}

From (17) we define

$$
\begin{aligned}
& H\left(s_{i}\right)=s_{i}^{5}+\left(6 h_{4}+3 h_{5}+2 \mu\right) s_{i}^{4}+\left(3 h_{5}^{2}+13 \alpha^{2}+15 h_{5}(\alpha+\lambda)+6 \beta \mu+4 \gamma \mu+(3 \lambda+\mu)\right. \\
& (5 \lambda+\mu)+\alpha(30 \lambda+8 \mu)) s_{i}^{3}+\left(12 \alpha^{3}+\beta^{3}+3 \beta^{2} \gamma+3 \beta \gamma^{2}+\gamma^{3}+12 \beta^{2} \lambda+24 \beta \gamma \lambda+12\right. \\
& \gamma^{2} \lambda+30 \beta \lambda^{2}+30 \gamma \lambda^{2}+19 \lambda^{3}+2\left(h_{3}+\gamma\right)(3 \beta+\gamma+6 \lambda) \mu+(3 \beta+\gamma+3 \lambda) \mu^{2}+2 \alpha^{2}(13 \beta \\
& \left.+13 \gamma+5(5 \lambda+\mu))+\alpha\left(12 h_{5}^{2}+60 h_{5} \lambda+57 \lambda^{2}+24 \lambda \mu+\mu(18 \beta+14 \gamma+3 \mu)\right)\right) s_{i}^{2}+\left(4 \alpha^{4}\right. \\
& +3\left(h_{3}+\gamma\right)^{2} \lambda\left(h_{5}+4 \lambda\right)+2 \mu\left(h_{5}^{2} \beta+4 \lambda^{3}+3 h_{5} \lambda(2 \beta+\gamma+3 \lambda)\right) \mu+\left(3 \beta^{2}+3 \lambda(\gamma+\lambda)+2\right.
\end{aligned}
$$




\section{Salah EL-Sherbeny, A. M. Rashad, D. M. Gharieb}

$$
\begin{aligned}
& \beta(\gamma+3 \lambda)) \mu^{2}+2 \alpha^{3}(9 \beta+9 \gamma+15 \lambda+2 \mu)+\alpha^{2}\left(15 \beta^{2}+15 \gamma^{2}+72 \gamma \lambda+63 \lambda^{2}+14 \gamma \mu\right. \\
& \left.+20 \lambda \mu+2 \mu^{2}+2 \beta(15 \gamma+36 \lambda+8 \mu)\right)+3 \alpha\left(\beta^{3}+\gamma^{3}+2 \gamma^{2}(6 \lambda+\mu)+\gamma(3 \lambda+\mu)(9 \lambda+\mu)\right. \\
& +2 \lambda\left(8 \lambda^{2}+4 \lambda \mu+\mu^{2}\right)+\beta\left(3\left(\gamma^{2}+8 \gamma \lambda+9 \lambda^{2}\right)+6(\gamma+2 \lambda) \mu+2 \mu^{2}\right)+\beta^{2}(3 \gamma+4(3 \lambda \\
& +\mu)))) s_{i}+\left(4 \alpha^{4}\left(h_{3}+\gamma\right)+3\left(h_{3}+\gamma\right)^{3} \lambda^{2}+\lambda \mu\left(\lambda^{2}(6 \beta+7 \gamma+2 \lambda)+2 h_{5}^{2}(\beta+3 \lambda)\right)+\left(\lambda^{3}\right.\right. \\
& \left.+h_{5}\left(\beta^{2}+3 \beta \lambda+3 \lambda^{2}\right)\right) \mu^{2}+2 \alpha^{3}\left(h_{3}+\gamma\right)\left(3 h_{5}+9 \lambda+2 \mu\right)+\alpha\left(3 \lambda\left(h_{3}+\gamma\right)^{2}\left(2 h_{5}+5 \lambda\right)\right. \\
& \left.+\mu\left(2 h_{5}^{2}(\beta+6 \lambda)+\lambda^{2}(18 \beta+21 \gamma+8 \lambda)\right)+3\left(\lambda^{2}+h_{5}(\beta+2 \lambda)\right) \mu^{2}\right)+2 \alpha^{2}\left(\beta^{3}+\gamma^{3}+3 \beta^{2}\right. \\
& (\gamma+4 \lambda+\mu)+2 \gamma^{2}(6 \lambda+\mu)+\lambda\left(13 \lambda^{2}+5 \lambda \mu+\mu^{2}\right)+\beta\left(3 \gamma^{2}+24 \gamma \lambda+24 \lambda^{2}+5 \gamma \mu\right. \\
& \left.\left.\left.+8 \lambda \mu+\mu^{2}\right)+\gamma\left(24 \lambda^{2}+9 \lambda \mu+\mu^{2}\right)\right)\right) \text {. } \\
& H_{1}=\left[\beta \mu \left(h_{1} h_{4}\left(h_{1}+h_{5}\right)\left(h_{3}+\gamma\right)\left(h_{3}+\alpha+\gamma\right)+h_{1}\left(h_{3}+\alpha+\gamma\right)\left(h_{5} h_{7}+\lambda\left(2 h_{6}+3 \gamma+\lambda\right)\right) \mu\right.\right. \\
& \left.\left.+\left(h_{5} h_{6} h_{7}+h_{1} h_{4} \lambda+3 h_{5}\left(h_{1}+\beta\right) \lambda\right) \mu^{2}\right)\right] \text {. } \\
& H_{1}\left(s_{i}\right)=\left[h _ { 1 } h _ { 4 } \beta \gamma ^ { 2 } \left(-\left(h_{2}+s_{i}\right)\left(h_{2}+s_{i}+\alpha\right)-\left(h_{2}+s_{i}\right)\left(h_{1}+s_{i}+\beta\right)-\left(h_{3}+s_{i}+\alpha\right)\left(h_{1}+\right.\right.\right. \\
& \left.\left.s_{i}+\beta\right)-\beta \gamma+h_{4} \mu\right)-h_{1} \beta \gamma^{2}\left(\left(h_{3}+s_{i}+\gamma\right)\left(s_{i}+\lambda\right)\left(h_{5}+2 s_{i}+3 \alpha+2 \lambda\right)+\left(3 s_{i}^{2}+h_{5} \beta+2\right.\right. \\
& \left.\left.\alpha \beta+2 s_{i}(\alpha+2 \beta+\gamma)+7 s_{i} \lambda+5 \alpha \lambda+4 \beta \lambda+3 \gamma \lambda+4 \lambda^{2}\right) \mu+\left(h_{10}+s_{i}\right) \mu^{2}\right)-h_{4}\left(s_{i}+\right. \\
& \beta)\left(s_{i}+\gamma+\mu\right)\left(-h_{1}\left(h_{3}+s_{i}\right)\left(\left(h_{3}+s_{i}+\alpha\right)\left(h_{1}+s_{i}+\beta\right)+\beta \gamma\right)-h_{1} \beta \gamma\left(h_{5}+2 s_{i}+3 \alpha+\right.\right. \\
& 2 \lambda+\mu))-h_{1} h_{4} \beta \gamma\left(\left(h_{3}+s_{i}\right)\left(\left(h_{3}+s_{i}+\alpha\right)\left(h_{1}+s_{i}+\beta\right)+\beta \gamma\right)+\beta \gamma\left(h_{5}+2 s_{i}+3 \alpha+2 \lambda\right.\right. \\
& \left.+\mu)-\lambda \mu\left(h_{5}+2 s_{i}+3 \alpha+2 \lambda+\mu\right)\right)+h_{1} \beta \gamma\left(-\left(h_{2}+s_{i}\right)\left(h_{3}+s_{i}\right)\left(( h _ { 3 } + s _ { i } + \alpha ) \left(h_{1}+s_{i}+\right.\right.\right. \\
& \beta)+\beta \gamma)+\beta \gamma\left(2 h_{3} s_{i}+s_{i}^{2}+3 s_{i} \alpha+2 \alpha^{2}+3 \alpha \beta+\beta^{2}+\beta \gamma+3 \alpha \lambda+2 \beta \lambda+\lambda^{2}-h_{4} \mu\right) \\
& \left.-\lambda \mu\left(2 h_{3} s_{i}+s_{i}^{2}+3 s_{i} \alpha+2 \alpha^{2}+3 \alpha \beta+\beta^{2}+\beta \gamma+3 \alpha \lambda+2 \beta \lambda+\lambda^{2}-h_{4} \mu\right)\right)+h_{1} h_{4}\left(s_{i}\right. \\
& +\beta) \gamma\left(-\left(h_{2}+s_{i}+\alpha\right) \lambda \mu+\left(\left(h_{3}+s_{i}+\alpha\right)\left(h_{1}+s_{i}+\beta\right)+\beta \gamma\right)\left(s_{i}+\gamma+\mu\right)+\left(s_{i}+\gamma+\mu\right)\right. \\
& \left.\left(\left(h_{2}+s_{i}\right)\left(h_{2}+s_{i}+\alpha\right)-h_{4} \mu\right)+\left(h_{1}+s_{i}+\beta\right)\left(-\lambda \mu+\left(h_{2}+s_{i}\right)\left(s_{i}+\gamma+\mu\right)\right)\right)-\left(s_{i}+\beta\right) \\
& \left(h_{1} \beta \gamma\left(s_{i}+\gamma+\mu\right)\left(2 h_{3} s_{i}+s_{i}^{2}+3 s_{i} \alpha+2 \alpha^{2}+3 \alpha \beta+\beta^{2}+\beta \gamma+3 \alpha \lambda+2 \beta \lambda+\lambda^{2}-h_{4} \mu\right)\right. \\
& \left.-h_{1}\left(h_{3}+s_{i}\right)\left(\left(h_{3}+s_{i}+\alpha\right)\left(h_{1}+s_{i}+\beta\right)+\beta \gamma\right)\left(-\lambda \mu+\left(h_{2}+s_{i}\right)\left(s_{i}+\gamma+\mu\right)\right)\right)-\left(s_{i}+\beta\right) \gamma \\
& \left(h_{1} \beta \gamma\left(s_{i}+\gamma+\mu\right)\left(h_{5}+2 s_{i}+3 \alpha+2 \lambda+\mu\right)-h_{1}\left(h_{3}+s_{i}\right)\left(-\left(h_{2}+s_{i}+\alpha\right) \lambda \mu+\left(s_{i}+\gamma+\mu\right)\right.\right. \\
& \left.\left.\left(\left(h_{2}+s_{i}\right)\left(h_{2}+s_{i}+\alpha\right)-h_{4} \mu\right)+\left(h_{1}+s_{i}+\beta\right)\left(-\lambda \mu+\left(h_{2}+s_{i}\right)\left(s_{i}+\gamma+\mu\right)\right)\right)\right)-\gamma(-\beta \gamma \\
& \left(\beta \gamma\left(\left(h_{2}+s_{i}+\alpha\right)\left(h_{3}+s_{i}+\alpha\right)-\beta \gamma+h_{4} \mu\right)-\lambda \mu\left(\left(h_{2}+s_{i}+\alpha\right)\left(h_{3}+s_{i}+\alpha\right)-\beta \gamma+h_{4} \mu\right)\right. \\
& \left.-\left(h_{3}+s_{i}\right)\left(-\left(h_{2}+s_{i}\right) \beta \gamma+\left(h_{3}+s_{i}+\alpha\right)\left(\left(h_{2}+s_{i}\right)\left(h_{2}+s_{i}+\alpha\right)-h_{4} \mu\right)\right)\right)-\left(s_{i}+\beta\right)(-\beta \gamma
\end{aligned}
$$




$$
\begin{aligned}
& \left(s_{i}+\gamma+\mu\right)\left(\left(h_{2}+s_{i}+\alpha\right)\left(h_{3}+s_{i}+\alpha\right)-\beta \gamma+h_{4} \mu\right)+\left(h_{3}+s_{i}\right)\left(-\beta \gamma\left(-\lambda \mu+\left(h_{2}+s_{i}\right)\left(s_{i}\right.\right.\right. \\
& \left.\left.\left.+\gamma+\mu))+\left(h_{3}+s_{i}+\alpha\right)\left(-\left(h_{2}+s_{i}+\alpha\right) \lambda \mu+\left(s_{i}+\gamma+\mu\right)\left(\left(h_{2}+s_{i}\right)\left(h_{2}+s_{i}+\alpha\right)-h_{4} \mu\right)\right)\right)\right)\right) \\
& +\left(h_{1}+s_{i}+\beta\right)\left(\beta \gamma \left(\beta \gamma\left(\left(h_{2}+s_{i}+\alpha\right)\left(h_{3}+s_{i}+\alpha\right)-\beta \gamma+h_{4} \mu\right)-\lambda \mu\left(( h _ { 2 } + s _ { i } + \alpha ) \left(h_{3}+\right.\right.\right.\right. \\
& \left.\left.s_{i}+\alpha\right)-\beta \gamma+h_{4} \mu\right)-\left(h_{3}+s_{i}\right)\left(-\left(h_{2}+s_{i}\right) \beta \gamma+\left(h_{3}+s_{i}+\alpha\right)\left(\left(h_{2}+s_{i}\right)\left(h_{2}+s_{i}+\alpha\right)-h_{4}\right.\right. \\
& \mu)))+\left(s_{i}+\beta\right)\left(-\beta \gamma\left(s_{i}+\gamma+\mu\right)\left(\left(h_{2}+s_{i}+\alpha\right)\left(h_{3}+s_{i}+\alpha\right)-\beta \gamma+h_{4} \mu\right)+\left(h_{3}+s_{i}\right)(-\beta \gamma\right. \\
& \left(-\lambda \mu+\left(h_{2}+s_{i}\right)\left(s_{i}+\gamma+\mu\right)\right)+\left(h_{3}+s_{i}+\alpha\right)\left(-\left(h_{2}+s_{i}+\alpha\right) \lambda \mu+\left(s_{i}+\gamma+\mu\right)\left(\left(h_{2}+s_{i}\right)\right.\right. \\
& \left.\left.\left.\left.\left.\left.\left(h_{2}+s_{i}+\alpha\right)-h_{4} \mu\right)\right)\right)\right)\right)\right] .
\end{aligned}
$$

From (31) we have

$$
\begin{aligned}
& E_{1}=\beta \mu\left(h_{8}\left(h_{5}+h_{8}\right)\left(h_{5}+2 \lambda\right)+\left(2 h_{8} \lambda+h_{5}\left(h_{6}+4 \lambda\right)\right) \mu\right) \text {. } \\
& E\left(s_{i}\right)=s_{i}^{3}+s_{i}^{2}\left(2\left(h_{6}+\gamma+4 \lambda\right)+\mu\right)+s_{i}\left(\alpha^{2}+\beta^{2}+\gamma^{2}+12 \gamma \lambda+20 \lambda^{2}+\gamma \mu+4 \lambda \mu+2 \beta\right. \\
& \left.(\gamma+6 \lambda+\mu)+\alpha\left(3 h_{5}+10 \lambda+\mu\right)\right)+\left(h_{7}+\gamma\right)\left(h_{7}+\alpha+\gamma\right)(\alpha+4 \lambda)+h_{5} h_{6} \mu+2\left(2 h_{5}+h_{8}\right) \lambda \mu \text {. } \\
& E_{1}\left(s_{i}\right)=s_{i}^{5}+\left(3 h_{5}+2(\alpha+4 \lambda+\mu)\right) s_{i}^{4}+\left(\alpha^{2}+3 \beta^{2}+3 \gamma^{2}+5 \alpha\left(h_{10}+\gamma\right)+20 \gamma \lambda+20 \lambda^{2}\right. \\
& \left.+3 \alpha \mu+4 \gamma \mu+10 \lambda \mu+\mu^{2}+\beta(6 \gamma+20 \lambda+6 \mu)\right) s_{i}^{3}+\left(\beta^{3}+3 \beta^{2} \gamma+3 \beta \gamma^{2}+\gamma^{3}+16 \beta^{2} \lambda\right. \\
& +32 \beta \gamma \lambda+16 \gamma^{2} \lambda+36 \beta \lambda^{2}+36 \gamma \lambda^{2}+16 \lambda^{3}+2\left(h_{5}+\lambda\right)(3 \beta+\gamma+8 \lambda) \mu+(3 \beta+\gamma+4 \lambda) \mu^{2} \\
& +\alpha^{2}\left(2\left(h_{5}+\lambda\right)+\mu\right)+\alpha\left(4 \beta^{2}+4 \gamma^{2}+18 \gamma \lambda+12 \lambda^{2}+5 \gamma \mu+10 \lambda \mu+\mu^{2}+\beta(8 \gamma+18 \lambda\right. \\
& +7 \mu)) s_{i}^{2}+\left(h_{5}\left(h_{5}+h_{8}\right)\left(h_{5}+2 \lambda\right)(\alpha+4 \lambda)+\left(2 h_{5}^{2} \beta+\alpha\left(2 h_{3} \alpha+5 h_{5} \beta+2 h_{5} \gamma+\alpha \gamma\right)+\right.\right. \\
& \left.2\left(\left(7 h_{5}+8 \alpha\right) \beta+4\left(h_{5}+\alpha\right) \gamma\right) \lambda+4(2 \alpha+6 \beta+3 \gamma) \lambda^{2}+8 \lambda^{3}\right) \mu+\left(2 h_{3} \alpha+\alpha \gamma+h_{10}(3 \beta\right. \\
& \left.+2(\gamma+\lambda))) \mu^{2}\right) s_{i}+\beta \mu\left(h_{8}\left(h_{5}+h_{8}\right)\left(h_{5}+2 \lambda\right)+\left(2 h_{8} \lambda+h_{5}\left(h_{6}+4 \lambda\right)\right) \mu\right) \text {. } \\
& Z(s)=s_{i}^{5}+s_{i}^{4}\left(6 \alpha+3\left(h_{5}+4 \lambda\right)+2 \mu\right)+s_{i}^{3}\left(3 h_{5}\left(h_{5}+5 h_{8}\right)+13 \alpha^{2}+6 \beta \mu+4 \gamma \mu+(6 \lambda+\mu)\right. \\
& (10 \lambda+\mu)+\alpha(60 \lambda+8 \mu))+s_{i}^{2}\left(12 \alpha^{3}+\beta^{3}+3 \beta^{2} \gamma+3 \beta \gamma^{2}+\gamma^{3}+24 \beta^{2} \lambda+48 \beta \gamma \lambda+24 \gamma^{2} \lambda\right. \\
& +120 \beta \lambda^{2}+120 \gamma \lambda^{2}+152 \lambda^{3}+2\left(h_{10}+\gamma\right)(3 \beta+\gamma+12 \lambda) \mu+(3 \beta+\gamma+6 \lambda) \mu^{2}+2 \alpha^{2}(13 \beta \\
& \left.+13 \gamma+50 \lambda+5 \mu)+\alpha\left(12\left(h_{5}^{2}+10 h_{5} \lambda+19 \lambda^{2}\right)+2(9 \beta+7 \gamma+24 \lambda) \mu+3 \mu^{2}\right)\right)+s_{i}\left(4 \alpha^{4}\right. \\
& +6\left(h_{10}+\gamma\right)^{2} \lambda\left(h_{5}+8 \lambda\right)+2\left(h_{5}^{2} \beta+32 \lambda^{3}+6 h_{5} \lambda(2 \beta+\gamma+6 \lambda)\right) \mu+\left(3 \beta^{2}+6 \lambda(\gamma+2 \lambda)\right. \\
& +2 \beta(\gamma+6 \lambda)) \mu^{2}+2 \alpha^{3}(9 \beta+9 \gamma+30 \lambda+2 \mu)+\alpha^{2}\left(15 h_{5}^{2}+144 h_{5} \lambda+2\left(126 \lambda^{2}+20 \lambda \mu\right.\right. \\
& +\mu(8 \beta+7 \gamma+\mu)))+3 \alpha\left(\beta^{3}+\gamma^{3}+2 \gamma^{2}(12 \lambda+\mu)+\gamma(6 \lambda+\mu)(18 \lambda+\mu)+4 \lambda\left(32 \lambda^{2}+\right.\right. \\
& \left.\left.\left.8 \lambda \mu+\mu^{2}\right)+\beta^{2}(3 \gamma+4(6 \lambda+\mu))+\beta\left(3 \gamma^{2}+6 \gamma(8 \lambda+\mu)+2\left(54 \lambda^{2}+12 \lambda \mu+\mu^{2}\right)\right)\right)\right)+
\end{aligned}
$$




\section{Salah EL-Sherbeny, A. M. Rashad, D. M. Gharieb}

$\left(4 \alpha^{4}\left(h_{10}+\gamma\right)+12\left(h_{10}+\gamma\right)^{3} \lambda^{2}+4 \lambda\left(2 \lambda^{2}(6 \beta+7 \gamma+4 \lambda)+h_{5}^{2}(\beta+6 \lambda)\right) \mu+\left(8 \lambda^{3}+h_{5}\right.\right.$ $\left.\left(\beta^{2}+6 \beta \lambda+12 \lambda^{2}\right)\right) \mu^{2}+\alpha\left(12\left(h_{10}+\gamma\right)^{2} \lambda\left(h_{5}+5 \lambda\right)+2\left(h_{5}^{2}(\beta+12 \lambda)+2 \lambda^{2}(18 \beta+21\right.\right.$ $\left.\gamma+16 \lambda)) \mu+3\left(\beta^{2}+4 \lambda(\gamma+\lambda)+\beta(\gamma+4 \lambda)\right) \mu^{2}\right)+2 \alpha^{3}\left(h_{10}+\gamma\right)\left(3 h_{5}+2(9 \lambda+\mu)\right)+2$ $\alpha^{2}\left(\beta^{3}+\gamma^{3}+3 \beta^{2}(\gamma+8 \lambda+\mu)+2 \gamma^{2}(12 \lambda+\mu) 2 \lambda\left(52 \lambda^{2}+10 \lambda \mu+\mu^{2}\right)+\beta\left(3 \gamma^{2}+48\right.\right.$ $\left.\left.\left.\gamma \lambda+96 \lambda^{2}+5 \gamma \mu+16 \lambda \mu+\mu^{2}\right)+\gamma\left(96 \lambda^{2}+18 \lambda \mu+\mu^{2}\right)\right)\right)$.

From (49), we have

$$
\begin{aligned}
& Z_{1}=\beta \mu\left(2 h_{4}\left(h_{11}+h_{5}\right) h_{8}\left(h_{5}+h_{8}\right)\left(h_{5}+2 \lambda\right)+2 h_{4}\left(h_{5}+h_{8}\right)\left(h_{5} h_{7}+2 \lambda\left(2 h_{6}+3 \gamma+2 \lambda\right)\right) \mu+\right. \\
& \left.\left(h_{5} h_{6} h_{7}+4 h_{4} h_{8} \lambda+6 h_{5}\left(h_{11}+\beta\right) \lambda\right) \mu^{2}\right) \text {. } \\
& Z_{1}\left(s_{i}\right)=2 h_{4} h_{8} \beta \gamma^{2}\left(-\left(h_{9}+s_{i}\right)\left(h_{10}+s_{i}+2 \alpha\right)-\left(h_{10}+s_{i}+2 \alpha\right)\left(h_{8}+s_{i}+\beta\right)-\beta \gamma+h_{8} \mu\right. \\
& \left.-\left(h_{9}+s_{i}\right)\left(h_{8}+s_{i}+\gamma+\mu\right)\right)-2 h_{4} h_{8}\left(s_{i}+\beta\right)\left(s_{i}+\gamma+\mu\right)\left(-\left(h_{10}+s_{i}\right)\left(( h _ { 1 0 } + s _ { i } + 2 \alpha ) \left(h_{8}\right.\right.\right. \\
& \left.\left.\left.+s_{i}+\beta\right)+\beta \gamma\right)-\beta \gamma\left(h_{5}+2 s_{i}+3 \alpha+4 \lambda+\mu\right)\right)-2 h_{4} h_{8} \beta \gamma\left(( h _ { 1 0 } + s _ { i } ) \left(( h _ { 1 0 } + s _ { i } + 2 \alpha ) \left(h_{8}\right.\right.\right. \\
& \left.\left.\left.+s_{i}+\beta\right)+\beta \gamma\right)+\beta \gamma\left(h_{5}+2 s_{i}+3 \alpha+4 \lambda+\mu\right)-2 \lambda \mu\left(h_{5}+2 s_{i}+3 \alpha+4 \lambda+\mu\right)\right)+2 h_{4} \beta \gamma \\
& \left(-\left(h_{10}+s_{i}\right)\left(h_{9}+s_{i}\right)\left(\left(h_{10}+s_{i}+2 \alpha\right)\left(h_{8}+s_{i}+\beta\right)+\beta \gamma\right)+\beta \gamma\left(s_{i}^{2}+3 h_{10} \alpha+2 \alpha^{2}+h_{5} \beta+\right.\right. \\
& \left.4 h_{3} \lambda+s_{i}(3 \alpha+2 \beta+4 \lambda)-h_{8} \mu\right)-2 \lambda \mu\left(s_{i}^{2}+3 h_{10} \alpha+2 \alpha^{2}+h_{5} \beta+4 h_{3} \lambda+s_{i}(3 \alpha+2 \beta\right. \\
& \left.\left.+4 \lambda)-h_{8} \mu\right)\right)-2 h_{4}\left(s_{i}+\beta\right) \gamma\left(\beta \gamma\left(s_{i}+\gamma+\mu\right)\left(h_{5}+2 s_{i}+3 \alpha+4 \lambda+\mu\right)-\left(h_{10}+s_{i}\right)\left(\left(s_{i}\right.\right.\right. \\
& +\gamma)\left(s_{i}+\gamma+2 \lambda\right)\left(h_{5}+2 s_{i}+3 \alpha+4 \lambda\right)+\left(s_{i}+\gamma\right)\left(5 s_{i}+5 \alpha+2 \beta+3 \gamma+8 \lambda\right) \mu+\left(h_{10}+4 s_{i}\right. \\
& \left.\left.+2 \alpha+3 \gamma) \mu^{2}+\mu^{3}\right)\right)-2 h_{4} h_{8}\left(s_{i}+\beta\right) \gamma\left(-\left(\left(h_{10}+s_{i}+2 \alpha\right)\left(h_{8}+s_{i}+\beta\right)+\beta \gamma\right)\left(s_{i}+\gamma+\mu\right)\right. \\
& +2 \lambda \mu\left(h_{8}+s_{i}+\gamma+\mu\right)-\left(s_{i}+\gamma+\mu\right)\left(h_{8}\left(s_{i}+\gamma+2 \lambda\right)+\left(h_{9}+s_{i}\right)\left(s_{i}+\gamma+\mu\right)\right)-\left(h_{10}+\right. \\
& \left.\left.s_{i}+2 \alpha\right)\left(-2 \lambda \mu+\left(h_{9}+s_{i}\right)\left(s_{i}+\gamma+\mu\right)\right)\right)-2 h_{4}\left(s_{i}+\beta\right)\left(\beta \gamma ( s _ { i } + \gamma + \mu ) \left(s_{i}^{2}+3 h_{10} \alpha+2 \alpha^{2}\right.\right. \\
& \left.+h_{5} \beta+4 h_{3} \lambda+s_{i}(3 \alpha+2 \beta+4 \lambda)-h_{8} \mu\right)-\left(h_{10}+s_{i}\right)\left(\left(h_{10}+s_{i}+2 \alpha\right)\left(h_{8}+s_{i}+\beta\right)+\beta \gamma\right) \\
& \left.\left(-2 \lambda \mu+\left(h_{9}+s_{i}\right)\left(s_{i}+\gamma+\mu\right)\right)\right)+2 h_{4} \beta \gamma^{2}\left(\beta \gamma\left(h_{5}+2 s_{i}+3 \alpha+4 \lambda+\mu\right)-2 \lambda \mu\left(h_{5}+2 s_{i}\right.\right. \\
& \left.+3 \alpha+4 \lambda+\mu)+\left(h_{10}+s_{i}\right)\left(-\left(h_{9}+s_{i}\right)\left(h_{10}+s_{i}+2 \alpha\right)+h_{8} \mu-\left(h_{9}+s_{i}\right)\left(h_{8}+s_{i}+\gamma+\mu\right)\right)\right) \\
& -\gamma\left(-\beta \gamma\left(\beta \gamma\left(\left(h_{8}+s_{i}\right)\left(h_{5}+h_{8}+s_{i}\right)+\left(h_{7}+s_{i}+4 \lambda\right) \mu\right)-2 \lambda \mu\left(\left(h_{8}+s_{i}\right)\left(h_{5}+h_{8}+s_{i}\right)+\right.\right.\right. \\
& \left.\left(h_{7}+s_{i}+4 \lambda\right) \mu\right)-\left(h_{10}+s_{i}\right)\left(-\left(h_{9}+s_{i}\right) \beta \gamma+\left(h_{8}+s_{i}+\beta\right)\left(h_{8}\left(s_{i}+\gamma+2 \lambda\right)+\left(h_{9}+s_{i}\right)\right.\right. \\
& \left.\left.\left.\left(s_{i}+\gamma+\mu\right)\right)\right)\right)-\left(s_{i}+\beta\right)\left(-\beta \gamma\left(s_{i}+\gamma+\mu\right)\left(\left(h_{8}+s_{i}\right)\left(h_{5}+h_{8}+s_{i}\right)+\left(h_{7}+s_{i}+4 \lambda\right) \mu\right)+\right. \\
& \left(h_{10}+s_{i}\right)\left(-\beta \gamma\left(-2 \lambda \mu+\left(h_{9}+s_{i}\right)\left(s_{i}+\gamma+\mu\right)\right)+\left(h_{8}+s_{i}+\beta\right)\left(-2 \lambda \mu\left(h_{8}+s_{i}+\gamma+\mu\right)+\right.\right. \\
& \left.\left.\left.\left.\left(s_{i}+\gamma+\mu\right)\left(h_{8}\left(s_{i}+\gamma+2 \lambda\right)+\left(h_{9}+s_{i}\right)\left(s_{i}+\gamma+\mu\right)\right)\right)\right)\right)\right)+\left(h_{10}+s_{i}+2 \alpha\right)\left(\beta \gamma \left(\beta \gamma \left(\left(h_{8}\right.\right.\right.\right.
\end{aligned}
$$




$$
\begin{aligned}
& \left.\left.+s_{i}\right)\left(h_{5}+h_{8}+s_{i}\right)+\left(h_{7}+s_{i}+4 \lambda\right) \mu\right)-2 \lambda \mu\left(\left(h_{8}+s_{i}\right)\left(h_{5}+h_{8}+s_{i}\right)+\left(h_{7}+s_{i}+4 \lambda\right) \mu\right)- \\
& \left.\left(h_{10}+s_{i}\right)\left(-\left(h_{9}+s_{i}\right) \beta \gamma+\left(h_{8}+s_{i}+\beta\right)\left(h_{8}\left(s_{i}+\gamma+2 \lambda\right)+\left(h_{9}+s_{i}\right)\left(s_{i}+\gamma+\mu\right)\right)\right)\right)+\left(s_{i}\right. \\
& +\beta)\left(-\beta \gamma\left(s_{i}+\gamma+\mu\right)\left(\left(h_{8}+s_{i}\right)\left(h_{5}+h_{8}+s_{i}\right)+\left(h_{7}+s_{i}+4 \lambda\right) \mu\right)+\left(h_{10}+s_{i}\right)(-\beta \gamma(-2 \lambda \mu\right. \\
& \left.+\left(h_{9}+s_{i}\right)\left(s_{i}+\gamma+\mu\right)\right)+\left(h_{8}+s_{i}+\beta\right)\left(-2 \lambda \mu\left(h_{8}+s_{i}+\gamma+\mu\right)+\left(s_{i}+\gamma+\mu\right)\left(h_{8}\left(s_{i}+\gamma+2 \lambda\right)\right.\right. \\
& \left.\left.\left.\left.\left.+\left(h_{9}+s_{i}\right)\left(s_{i}+\gamma+\mu\right)\right)\right)\right)\right)\right) .
\end{aligned}
$$

\section{References}

1. Chandrasekhar, P., Natarajan, R., and Yadavalli, V. S. S. (2004). A Study on a two unit standby system with Erlangian repair time. Asia-Pacific Journal of Operational Research, 21, 271-277.

2. Dhillon, B. S. and Rayapati, S. N. (1985). Stochastic analysis of two-unit outdoor electric power systems in changing weather. Microelectronics and reliability, 25, 357-367.

3. Khaled, M. EL-Said. and Mohamed, S. EL-Sherbeny. (2006). Comparing of reliability characteristic between two different systems. Applied Mathematics and Computation, 173, 1183-1199.

4. Wang, K.-H. and Pearn, W.-L. (2003) Cost benefit analysis of series systems with warm standby components. Mathematical Methods of Operations Research, 58, 247-258.

5. Wang, K.-H. and Sivazlian, B. D. (1997). Life cycle cost analysis for availability system with parallel components. Computers and Industrial Engineering, 33, 129- 132.

6. Wang, K.-H., Dong, W.-L., and Ke, J.-B. (2006) Comparison of reliability and the availability between four systems with warm standby components and standby switching failures. Applied Mathematics and Computation, 183, 1310-1322.

7. Wang, K.-H., Hsieh, C.-H., and Liou, C.-H. (2006). Cost benefit analysis of series systems with cold standby components and a repairable service station. Quality Technology \& Quantitative Management, 3, 77-92. 